\title{
Arbor
}

\section{La ciudad de Salamanca en el siglo XVI: La conjunción del arte y la ciencia}

\section{Cirilo Flórez Miguel}

Arbor CLXXIII, 683-684 (Noviembre-Diciembre 2002), 429-458 pp.

\section{La ciudad de Salamanca}

Una de las caracterísitcas más evidentes de esta ciudad es su «forma circular», que viene determinada por la muralla que desde antiguo establece el perímetro de la ciudad. La muralla salmantina hace de la ciudad de Salamanca una «ciudad circular», organizada en torno a un centro, el consistorio, que en siglo XVI estaba situado en la plaza de San Martín, próxima a lo que hoy es la plaza Mayor y correspondiéndose más o menos con lo que hoy es la plaza del Corrillo. Así, pues, la plaza de San Martín es el centro en torno al cual se organiza la ciudad, que en ese siglo XVI, está dejando de ser un «espacio agregado» de casas dentro del cual sobresalen las "torres fortaleza»; y está pasando a ser un «espacio sistema» gracias a la transformación que está protagonizando su urbanismo en ese siglo XVI con la modificación del edificio de la Universidad, la construcción de las principales «casas-palacios», así como la Iglesia de San Esteban junto al Convento de los dominicos y el mismo Convento de las Dueñas, vecino a aquel; la construcción del Colegio de Fonseca; y en el ámbito religioso la construcción de la «nueva Catedral», que andando el tiempo será otro de los grandes edificios de la ciudad ${ }^{1}$.

El viejo «espacio agregado» medieval se transforma en un «espacio sistema» y confiere a la ciudad de Salamanca una "forma simbólica», que va a mantenerse hasta la transformación que experimentará la ciudad a partir del final del siglo XX. El objeto de nuestro artículo 
es relatar cómo en el siglo XVI tiene lugar en la ciudad de Salamanca una revolución en la idea de espacio, que se hace manifiesta en dos de los objetos que según Cassirer son la expresión más clara de las transformaciones que tienen lugar en le Renacimiento: el arte y la ciencia. En la ciudad de Salamanca del siglo XVI se produce una transformación en la idea de espacio, que nos permite afirmar que en ese siglo la ciudad del Tormes logra una síntesis entre la cultura humanista (arte) y la cultura científica (ciencia), que es la que va a modelar su carácter de "ciudad renacentista» ${ }^{2}$.

Esa transformación de la idea de espacio está protagonizada por las dos élites más importantes de la ciudad de aquel momento: la élite de los nobles y caballeros y la élite de los hombres de letras. Veamos cómo tiene lugar esa transformación en torno a cada una de esas élites y de sus respectivas mentalidades. La primera de las transformaciones tiene lugar en el cambio de la «casa fortaleza» por la «casa palacio» y está protagonizada por la élite de los nobles y caballeros, aspecto éste que ha sido muy bien estudiado por Clara Isabel López Benito, a cuya obra remitimos ${ }^{3}$.

\section{La casa de la nobleza como forma simbólica}

El elemento más destacado de la nobleza desde el punto de vista de la representación es «la casa», que es el símbolo que mejor manifiesta ante los otros la relevancia del linaje dentro del que se encuentra el «hombre noble». La casa es la pieza básica que proyecta hacia el exterior, hacia los otros el status de la familia. Y en la casa la fachada es el signo en el que se concentra mejor esa dimensión de la casa noble. De ahí que en el siglo XVI asistamos en la ciudad de Salamanca a una significativa transformación de las casas de la nobleza. "La Oligarquía salmantina de la temprana Edad Moderna se caracterizaba por ser esencialmente urbana. Su casa principal estaba por tanto ubicada en la Ciudad, hecho que sin embargo no impedía que los miembros de aquella mantuvieran además casa abierta en sus posesiones rurales» ${ }^{4}$.

Otro signo de distinción de las casas nobles es su localización, que en la ciudad de Salamanca tiene lugar en torno a la Plaza Pública y a dos de las parroquias de mayor renombre: la de San Benito y la de Sto Tomé de los caballeros. Un ejemplo muy claro de lo que venimos diciendo son las casas del linaje de los Maldonado que ocupaban un semicírculo que podemos trazar desde el hoy Museo Provincial ( Palacio de los Abarca Alcaraz) hasta el Convento de las Claras ( Palacio del 
comunero Pedro Maldonado) y Casa de las Conchas (Casa del Dr. Rodrigo Maldonado de Talavera). Dentro de ese semicírculo tenemos que incluir el espacio que hoy ocupa la Universidad Pontificia (antigua Casa de la Compañía de Jesús levantada en terrenos de otras casas de los Maldonado).

La transformación más importante experimentada por las casas de la nobleza salmantina en los siglos XV y XVI fue su metamorfosis de casa-fuerte, paradigma de una mentalidad medieval, en casa-palacio de estilo isabelino y que podemos interpretar como paradigma de una mentalidad renacentista. Los dos ejemplos más representativos de esto último son la Casa de las Conchas y la de los Abarca-Alcaraz (hoy Museo Provincial). El rasgo que las caracteriza es la rica decoración exterior organizada en torno a la fachada y las ventanas, que podemos relacionar con la afirmación de la propia individualidad, que es muy característico de la mentalidad renacentista.

Este rasgo de afirmación de la propia individualidad es algo que se aprecia muy bien en las dos élites que protagonizan la vida en la ciudad de Salamanca en su siglo dorado (el XVI): la élite de los nobles y la élite de los hombres de letras. Así como hemos visto que el elemento más característico de la nobleza desde el punto de vista de la representación es la casa, en el caso de los hombres de letras es el libro, que también tiene su portada, en la cual muchos hombres de letras han querido afirmar su individualidad imprimiendo su divisa o impresa; la cual es a los hombres de letras lo que la fachada de la casa a los hombres nobles ${ }^{5}$.

\section{Nominalismo y ciencia}

Otro lugar clásico para ver la transformación de la idea de espacio en la Salamanca del siglo XVI es el nominalismo y su presencia en dicha Universidad. Es un tema que ha sido muy deformado por algunos historiadores que lo han tratado tanto en relación a la Universidad de Salamanca como a la de París. Beltrán de Heredia hace de dicha tradición una de las grandes desgracias padecidas por la Universidad de Salamanca en la primera mitad del siglo XVI ${ }^{6}$. Pero no se trata de ninguna desgracia, sino de una de las tradiciones a las que cabe atribuir la relevancia de esta Universidad en el siglo XVI. La misma consideración negativa merece el nominalismo en la obra de Villoslada sobre la Universidad de París ${ }^{7}$. Una de las fuentes de este juicio 
negativo se encuentra en el juicio que merecen a Luis Vives los dialécticos de París ${ }^{8}$.

Los estudios que hoy se hacen sobre este grupo de la Universidad de París hacen una valoración muy distinta; resaltando sobre todo la importancia del grupo para la nueva física, tal como la misma se constituirá a partir de Galileo ${ }^{9}$. Este es el punto que nosotros queremos resaltar en nuestro estudio, fijando la atención en la importancia que tiene esa parte de este grupo, que ejerció como docente en la Universidad de Salamanca sobre todo a partir del año 1516 con la llegada de Silíceo a Salamanca, aunque ya se aprecian los intentos de renovación a partir de 1509.

Podemos iniciar esta tradición en la Universidad de Salamanca con el filósofo y teólogo Juan de Oria, que el año de 1509 se hace cargo de la cátedra de Scoto, así como de la de lógica y filosofía de nominales; en cuya enseñanza permanece hasta el curso 1518-19 en el que pasa a la cátedra de Biblia, en la cual se va a encontrar con los problemas que le trajeron la condena de la Inquisición. A nosotros nos interesa su estudio como filósofo nominal.

El año de 1518 publica una repetición: Sobre la inmortalidad del alma, que era uno de los temas a debate en la filosofía europea del momento. Discute las opiniones de distintos autores y mantiene una propia que se encuentra dentro de la ortodoxia católica. Lo más significativo para nuestro estudio es su tratamiento de las potencias del alma, que no las considera como algo accidental al modo de Sto Tomás, sino formando parte del alma como potencia operativa, que el hombre como viator tiene que actualizar. La fuerza operativa del alma exige un cuerpo que le corresponda y le sea adecuado, lo cual va a caracterizar la individuación del acto de pensar, cosa que no estaba dentro de la doctrina averroista acerca del entendimiento. Oria, en cambio, considera que el alma está incluida en el cuerpo como su morada exterior. Y al interpretar así al alma se inserta dentro de la tradición cristiana que tiene a San Agustín a su cabeza y que resalta el principio de la subjetividad, que es el que está en la base de lo que andando el tiempo será el idealismo de Descartes del que se hace partir la filosofía moderna. Y para ello juega un importante papel la educación y en concreto la enseñanza de la lógica como dialéctica, que es uno de los aspectos a destacar de Juan de Oria como maestro de lógica y filosofía nominal. Podemos relacionar las teorías de Oria en este punto con uno de los programas iconográficos de la Universidad de Salamanca: el programa iconográfico de la escalera, que hay que leer como el camino del homo viator hacia su perfección como hombre sabio por medio del adecuado cultivo de las facultades del alma. 
La ciudad de Salamanca en el siglo XVI: La conjunción...

\section{Nominalismo y física dinámica}

En los primeros años del siglo XVI tuvo lugar en la Universidad de París un renacimiento del interés por problemas de la física. Este interés fue suscitado por el nominalista escocés Juan Mair. Pero fueron sus discípulos los que escribieron un buen número de Quaestiones sobre la física aristotélica en las que en torno al movimiento local crearon una nueva física como resultado de la síntesis del pensamiento mertoniano proveniente de los calculadores de Oxford y de los seguidores en París de las doctrinas de Juan Buridán acerca del aumento y disminución de las cualidades. Dentro de los seguidores parisinos de Juan de Buridán nos encontramos con Nicolás de Oresme, que el año 1351 compuso un tratado titulado: Tractatus de configurationibus qualitatum et motum, que es el que a nosotros nos va a servir aquí como referencia para lo que queremos decir. El núcleo de este texto es su teoría de la configuración de la que podemos encontrar antecedentes en Aristóteles, Rogelio Bacon, Juan de Buridán y el oxfordiano Ricardo Swineshead.

Oresme expone su teoría de la configuración como un medio para la medición de las cualidades, cosa que puede hacerse imaginando la variación de la cualidad como representada por dos líneas: la de la extensión de la cualidad (longitud) y la de la intensidad de la cualidad (latitud). Este procedimiento de «imaginar» figuras geométricas para la medición de la variación de las «cualidades» de las cosas es lo que permite comprender el concepto central de esta teoría que es el de «uniformidad diforme», que luego será aplicado dentro de esta tradición a la medida del movimiento.

La nueva física parisina del siglo XVI tiene como una de sus caracterísitcas el aumento de las cuestiones experimentales y de la ejemplificación de los problemas tratados, lo que va a preparar el camino para la asociación del movimiento uniformemente diforme con la caida de los graves tal como será planteada por Galileo. En este terreno una serie de profesores ibéricos presentes en París van a formar lo que Wallace ha llamado el "eslabón ibérico», que nos conduce de París a Galileo pasando por la Península ibérica. En el inicio de ese eslabón ibérico nos encontramos con el portugués Alvaro Tomás, que escribió un tratado titulado Liber de triplice motu inspirado fundamentalmente en el oxfordiano Swineshead. Este eslabón será continuado en Salamanca por Pedro Margalho, Juan Martínez Silíceo y Domingo de Soto, que será el que acabe formulando la caida de los graves de modo similar a como lo hará definitivamente Galileo. Más adelante desarrollaremos pormenorizadamente este punto. 


\section{Silíceo y la física de Oxford y París en Salamanca}

El año 1516 llega Silíceo a la Universidad de Salamanca para regentar la cátedra de nominales en la Facultad de Artes. Esta llegada hay que entenderla dentro de la política global de renovación que la Universidad de Salamanca está llevando a cabo bajo la atenta mirada de los Reyes Católicos. Las primeras cátedras que se renovaron fueron la de Gramática, Poética y Retórica; y ahora toca el turno a las cátedras de Lógica y Filosofía Natural, que son de las más importantes de la Facultad de artes. Y esta misión la emprende Silíceo, que durante sus años en la Universidad de Salamanca va a jugar un papel decisivo. Al año de llegar a Salamanca es ya colegial del más importante Colegio Mayor de su Universidad: el de San Bartolomé. Y en los primeros años de su estancia en esta Universidad lleva a cabo una actividad productiva extraordinaria. Publica su Dialéctica o Lógica magna, que consta de cuatro partes de las que se conserva la primera y parte de la tercera. Publica también las Quaestiones al Perihermeneias de su maestro Dullaert de Gante, el libro de Suisseth (Ricardo de Swineshead) ${ }^{10}$ perteneciente a la rama oxfordiana de los calculadores y una Lógica brevis. Es decir, que pone a disposción de alumnos y profesores de la Universidad de Salamanca las doctrinas más actuales acerca de la lógica nominalista y de la filosofía natural tal como en ese momento se estaban practicando en la Universidad de París, que era la más importante del momento. Con la llegada de Silíceo a Salamanca la Facultad de artes logra uno de sus mejores momentos de la historia. Tiene una importante cátedra de Astrología, de Gramática, de Poética, de Retórica, de Lógica y de Filosofía natural. En todas ellas se enseñan las doctrinas más avanzadas del momento. Este auge en la Facultad de Artes coincide con la reforma del edificio, que podemos situar entre 1512 y 1528 , reforma dentro de la cual se construye el antepecho de la bibliteca, la escalera y la fachada de la Universidad, en cuya decoración queda también reflejado el auge de la Facultad de Artes y las ideas que circulan por las cátedras antes mencionadas.

El año de 1522 saca la cátedra de filosofía de la naturaleza que regentará hasta su marcha de Salamanca como obispo de Cartagena. En dicha cátedra se leían textos de Aristóteles y textos de matemática. Entre las obras que publica después de su llegada a Salamanca encontramos Calculatoris Suiset, obra mencionada en la nota anterior. Se trata de la obra del inglés Ricardo Swineshead, filósofo del siglo XIV que era llamado específicamente el Calculator, que pertenece al 
grupo de los calculadores de Oxford, que en el caso de la filosofia natural son los primeros que aplican la matemática al estudio del movimiento fundando la ciencia de la cinemática, que analiza el movimiento en términos de distancia y tiempo. Para este tipo de análisis se desarrollaron dos métodos. El primero de ellos es el del álgebra de palabras empleado por Bradwardino en Oxford, que fue seguido por el grupo del Merton College entre los que se encuentra Ricardo de Swineshead. Pero este grupo de los calculadores de Oxford no se interesó por los aspectos dinámicos del movimiento. $\mathrm{Y}$ fue con los dialécticos de París del siglo XVI donde el método de Bradwardino fue desarrollado en el contexto de una teoría dinámica de la física. Es en este contexto de París donde se estudia la llamada «latitud de las formas», que expresaba los grados en que una cualidad o «forma» aumentaba o disminuía numéricamente en relación a una escala fijada de antemano. Esta relación entre la intensio y la extensio es lo que va a dar lugar al nacimiento del segundo método de análisis para expresar las relaciones funcionales; se trata de un método geométrico por medio de gráficos. Siguiendo este método se hicieron descripciones cinemáticas de varias formas de movimiento, siendo una de ellas la que iba a manifestarse como teniendo una significación particular, por ser la que iba a conducir más tarde al análisis galileano del movimiento.

La Regla de la velocidad media del Merton College podemos exponerla tal como había sido demostrada geométricamente por Nicolás de Oresme en su obra: Tractatus de configurationibus qualitatum et motuum. En el capítulo 7 de la parte tercera Oresme formula así la Regla del Merton College: «Toda cualidad, si es uniformemente diforme, es de la misma cantidad como sería la cualidad de un sujeto similar o igual, que informara uniformemente a este sujeto según el grado del punto medio. Yo entiendo esto (según el grado del punto medio) como si la cualidad fuera lineal». Y para demostrar geométricamente esta regla construye una figura geométrica, que podemos aplicar al texto de Soto y que Oresme formula de la siguiente forma: "Sea una cualidad que puede ser representada por un triángulo ABC. Es una cualidad uniformemente diforme que, en el punto B, se hace igual a cero. Sea $\mathrm{D}$ el punto medio de la línea que representa al sujeto; el grado de intensidad que afecta a este punto está representado por la línea DE. La cualidad que tendría en todas sus partes el grado así determinado puede ser representada por el cuadrilátero AFGB... Además es evidente por la proposición 26 del libro I de los Elementos de Euclides que los dos triángulos EFC y EGB son iguales. Y por lo tanto el 
triángulo $\mathrm{ABC}$, que repesenta la cualidad uniformemente diforme, y el cuadrilátero AFGB, que representa la cualidad uniforme, según el grado del punto medio, son entonces iguales. Y las dos cualidades que pueden ser representadas, una por el triángulo y otra por el cuadrilátero, son entonces también iguales una a otra, que es lo que se había propuesto demostrar» ${ }^{11}$.

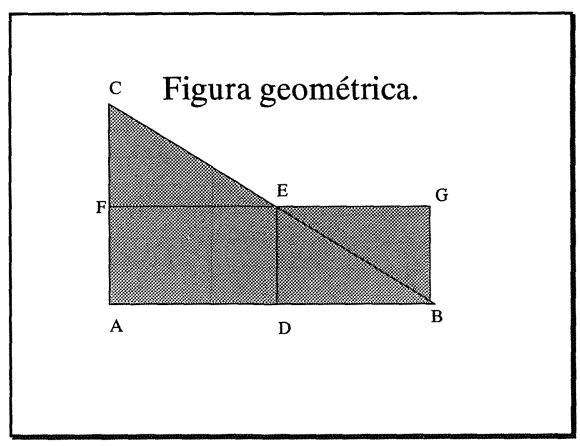

El grupo de los calculadores de Oxford y entre ellos Ricardo Swineshead lograron establecer la denominada Regla de la Velocidad Media del Merton College, consiguiendo además una demostración aritmética de la misma. Los calculadores del Merton College gozaron de gran popularidad en París; y de allí fueron importadas sus enseñanzas a Salamanca por Silíceo.

\section{La Lógica según la via de los reales y la de los nominales}

El año 1517 el protugués Pedro Margalho se incorpora a la Universidad de Salamanca procedente del Colegio Mayor de Santa Cruz de Valladolid para hacerse cargo de una cátedra de lógica de reales, cátedra que desempeñará hasta 1522. Tenemos, pues, que los dos catedráticos de lógica en la Facultad de artes en esos años posteriores a 1517 son Silíceo en la cátedra de nominales y Margalho en la de reales.Hemos visto que el año de 1517 Silíceo publica en Salamanca una obra sobre Dialéctica; y el año de 1520 publica Margalho también en Salamanca un texto de Lógica titulado: Logices utriusque scholia in divi Thomae subtilisque Duns doctrina ac nominalium. El texto de Margalho es, pues, una exposición de la Lógica de acuerdo a las tres vias: Santo Tomás, Scoto y los nominales. 
Tanto Silíceo como Margalho proceden de la Universidad de París y han venido a enseñar a la de Salamanca. Y lo que enseñan es precisamente la «dialéctica» que identifica a la escuela de París ${ }^{12}$. A este respecto es muy interesante la dedicatoria del libro de Margalho a Teodosio, hijo del gran Duque de Portugal. Y decimos que es interesante porque esta dedicatoria muestra muy bien el estado del saber en la Facultad de Artes de Salamanca en torno a esos años de 1520. La dedicatoria parte del bivium o doble camino que se le presenta al viajero cuando llega a una encrucijada. Margalho considera que el hijo del gran Duque de Portugal ha llegado a ese punto de su vida en el que el camino se divide en dos: uno que conduce a la sombra y a la noche profunda del Erebo y el otro que conduce a los Campos Elíseos. Y le recuerda que llegará a la edad perfecta cuando haya logrado la virtud completa; para lo cual le anima a seguir el «camino marcial» del espíritu combinando en su ingenio el testimonio de las musas (las buenas letras) y los ejercicios dialécticos de la escuela de París. Precisamente lo que pretende el libro de Margalho es enseñarle esos buenos ejercicios dialécticos, ya que «la lucha aguza el ingenio, perfecciona la lengua, vigoriza la voz y hace indeleble la memoria» ${ }^{13}$.

Este texto de Margalho es un elogio de la dialéctica, que aquí describe como una «lucha»; y que coloca al lado de las otras artes del lenguaje, afirmando que la virtud perfecta está en el cultivo de ambas líneas: la de las musas y la dialéctica. Esta dedicatoria de Margalho adquiere mayor relevancia si tenemos en cuenta que en el año en que la escribe acaba de construirse más o menos la famosa escalera de la Universidad de Salamanca, que hace alusión a este mismo tema. Podemos, pues, pensar en la escalera de la Universidad de Salamanca como un referente de lo que aquí está diciendo el protugués. Si pensamos en el ambiente cultural que en este momento impregna la Universidad de Salamanca, quizá no nos parezca exagerada la comparación que a continuación hace Margalho.

Hoy, escribe Margalho, la dialéctica parisiense como en otro tiempo la sabiduria de Atenas es objeto de la admiración de los grandes poetas. Y llega a afirmar retóricamente que en esos días en los que él escribe esa dedicatoria el saber de Atenas ha pasado a París y de París a Salamanca. Es uno de los elogios más extraordinarios que podemos oir de la Universidad de Salamanca en esos primeros años del siglo XVI. A parte de la retórica de lo que el elogio supone, lo cierto es que traduce un determinado estado de ánimo con respecto a la situación de la Facultad de Artes de la Universidad de Salamanca en las primeras décadas del siglo XVI ${ }^{14}$. 


\section{La docencia de Silíceo y los orígenes del camino moderno del filosofar}

La importancia de las enseñanazas de Silíceo en el terreno de la filosofía natural pueden también apreciarse por otro camino. Para ello vamos a fijar nuestra atención en un texto de un discípulo de Silíceo publicado el año 1554, cuando Silíceo es ya Arzobispo de Toledo y ha publicado su último libro: De divino nomine Jesus. El libro al que nos referimos es de Gómez Pereira, médico de Medina del Campo, y lleva por título: Antoniana Margarita ${ }^{15}$. El libro está dedicado a Silíceo y en esta dedicatoria el autor del libro escribe que Silíceo fue su maestro y preceptor en los conocimientos físicos, y que el contenido de la obra que le dedica es más suya (de Silíceo) que del propio autor.En la primera edición del texto aparece en la portada la «impresa» de Silíceo, lo que nos da a entender que el libro no solamente está dedicado a Silíceo, sino que además ha sido publicado bajo su protección. Lo que podemos interpretar como que Silíceo tiene un cierto compromiso con los contenidos del texto, que efectivamente consideraría como un desarrollo de sus enseñanzas durante la época en la que fue profesor de «físicos» de la Universidad de Salamanca.

El texto de Gómez Pereira, discípulo de Silíceo, es de una gran relevancia para comprender el modo como la modernidad va a plantear la filosofía. Este texto es un claro antecedente del planteamiento cartesiano; y tiene como peculiaridad el afirmar la autonomía del pensamiento en el acto de conocer. Gómez Pereira plantea por primera vez la originalidad del pensar, que aunque necesite del cuerpo para iniciarse como proceso, tiene en sí mismo una peculiaridad que le hace autónomo con respecto al cuerpo en el que se encuentra. Gómez Pereira explica el proceso de conocer de la siguiente manera: los objetos o fantasmas despiertan al alma, como consecuencia de esto el alma conoce que conoce algo y puesto que sabe que conoce algo, conoce a su vez que ella existe, ya que ella se conoce como distinta de aquello que ella conoce. Las palabras exactas del autor son: "Conozco que conozco algo; y aquel que conoce es, luego yo soy» («nosco me aliquid noscere, et quidquid noscit est, ergo ego sum») ${ }^{16}$. Gómez Pereira está planteando aquí la idea que en el siglo XVII formulará Descartes en su famosa expresión: pienso, luego existo. Tenemos, pues, que este texto de Gómez Pereira está indicándonos el camino por el que la filosofía moderna se desarrollará. Y ese camino no es otro que el de la dialéctica tal como fue planteada «al modo de París» y que tiene en Silíceo uno de sus más claros representantes. No exageramos, pues, 
al afirmar que las enseñanzas físicas de Silíceo en la Universidad de Salamanca están roturando un camino que más tarde encontrará su reconocimiento en el planteamiento cartesiano. Y en ese camino abierto por Silíceo entre otros el texto de Gómez Pereira juega un papel decisivo.

\section{Domingo de Soto como eslabón ibérico de la moderna dinámica}

En su obra Super octo libros phisicorum Aristotelis Quaestiones de 1552 describe Domingo de Soto la caida de los graves aplicando el concepto de «uniformidad diforme» de la siguiente forma: «El movimiento uniformemente diforme en cuanto al tiempo es aquel de tal modo diforme que, si se divide según el tiempo (es decir, según lo anterior y lo posterior), el movimiento del punto medio de cualquier parte supera al del extremo más lento en la misma proporción que es superado por el más intenso». Esta regla general del movimiento «uniformemente diforme» dice Domingo de Soto que es el tipo de movimiento característico de las cosas que se mueven naturalmente y de los proyectiles, cosa que confirma de la siguiente forma: «En efecto, cuando un cuerpo cae desde lo alto a través de un medio uniforme, se mueve al final más velozmente que al principio, mientras que el movimiento de los proyectiles es más lento al final que al principio, de tal manera que el primero aumenta y el segundo disminuye de modo uniformemente diforme... De todo esto, resulta que la velocidad del movimiento uniformemente diforme en cuanto al tiempo hay que estimarla mediante el grado medio y denominarla por él» ${ }^{17}$. Este texto de Domingo de Soto puede ser explicitado aplicando a la comprensión del mismo la figura geométrica que hemos expuesto anteriormente en la página 434. Lo que a nosotros nos interesa resaltar es el lugar que ocupan en relación con la historia de la ciencia moderna este autor y la Universidad de Salamanca en la tradición a la que nos hemos venido refiriendo. Para ello vamos a centrar nuestra atención en una polémica que ha tenido lugar entre algunos historiadores de la ciencia.

\section{La polémica acerca de los orígenes de la dinámica moderna}

La física matemática tal como será configurada por Galileo en el siglo XVII tiene sus antecedentes históricos reconocidos por todos los historiadores de la ciencia en los calculadores de Oxford del siglo XIV

y en la aplicación que en ese mismo siglo XIV se hizo en la Universidad 
de París de la matemática a la física. El resultado de estas aplicaciones y reflexiones fue la física parisina del XVI, que es el antecedente más inmediato de la física matemática tal como será formulada por Galileo. Los historiadores de la ciencia se han preguntado cómo llegaron las ideas de los calculadores de Oxford y París a Galileo. La respuesta a esta pregunta ha generado una polémica entre algunos historiadores de la ciencia. Duhem defiende que Soto es la fuente inmediata de los primeros escritos de Galileo ${ }^{18}$. Frente a esta tesis de Duhem Koyré defiende que Soto fue un escolástico español alejado totalmente del flujo principal del pensamiento europeo, que no pudo influir en Galileo ${ }^{19}$. El último paso en esta polémica lo ha dado Wallace, que considera que los jesuitas del Colegio Romano son los intermediarios entre Soto y Benedetti por un lado y Galileo por otro ${ }^{20}$.

Podemos aceptar la propuesta de Wallace, que ha logrado fijar muy bien los términos de la cuestión. Pero nosotros no vamos a fijarnos tanto en el «eslabón ibérico» entre los calculadores y Galileo; sino en la transformación que experimenta el concepto de movimiento local tal como fuera planteado por Aristóteles con las innovaciones que introducen los calculadores tanto de Oxford como de París; y cómo esas innovaciones están presentes en la Universidad de Salamanca en el siglo XVI.

El mérito de Soto en el texto anteriormente citado reside en el hecho de asociar el movimiento «uniformiter difformis» de los calculadores con la caida de los graves y mantener que la distancia de la caida puede ser calculada temporalmente sirviéndose del teorema mertoniano de la velocidad media. Y esta asociación no es algo casual y fortuito tal como piensa Koyré, sino el resultado de una serie de teorias y de estudios experimentales a lo largo de los siglos XIV, XV y XVI, que proporcionan el «trasfondo" del que va a surgir la formulación galileana de la caida de los graves y su nueva teoría del movimiento.

El punto de partida de las transformaciones acerca de la teoría del movimiento podemos situarlo en los trabajos de Thomas Bradwardine, que adquirieron renombre a través de los escritos de dos de sus discípulos: William Heytesbury y Richard Swineshead ${ }^{21}$. El cambio más importante introducido por estos autores se refiere a la intensificación de los cambios y de los movimientos. $\mathrm{Y}$ al aplicar esos cambios al movimiento local el término «intensificación» llega a ser sinónimo de velocidad; y los términos de «uniforme» $\mathrm{y}$ «diforme» pasan a asumir un significado cinemático.

Los calculadores de la Universidad de Oxford centraron sus estudios sobre el movimiento en la definición en abstracto de los distintos tipos de movimiento con términos matemáticos, pero sin ilustrar esos tipos de movimiento con ejemplos y experimentos tomados del universo físico. 
El paso hacia delante en el tratamiento del tema del movimiento lo dieron Alberto de Sajonia y Nicolás de Oresme en la Universidad de París en el siglo XV al ilustrar los diversos tipos de movimiento con ejemplos o experimentos tomados del universo físico. Estos filósofos de la Universidad de París se sirvieron del siguiente esquema:

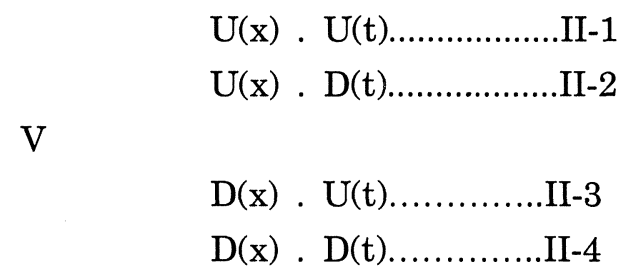

Los casos II,1, II,2 y II,4 los ejemplificó Nicolás de Oresme con la caida de un grave, introduciendo la variación de la velocidad en el movimiento local ${ }^{22}$.

En el siglo XV nos encontramos con Gaetano de Thiene, encuadrado en la tradición de Pablo de Venecia, que va a continuar las enseñanzas de los escolásticos de París del siglo XIV.

$\mathrm{Y}$ como culminación de todo este proceso que se iniciara en Oxford tiene lugar en la Universidad de París a principios del siglo XVI un creciente interés por los problemas de la física sobre todo bajo la influencia del nominalista escocés Juan Mair, muchos de cuyos discípulos escribieron importantes comentarios y cuestiones (Quaestiones) a la física aristotélica. En estos comentarios se produce la fusión entre el pensamiento de los calculadores de Oxford y los nominalistas parisinos del siglo XIV produciéndose una revolución en el campo de la física matemática del siglo XVI, que tiene su ubicación en la Universidad de París. Muchos estudiantes ibéricos que por esos años se encontraban en la Universidad de París son testigos de esta revolución; y van a ser ellos precisamente los que van a importar a España y Portugal y más en concreto a la Universidad de Salamanca esos desarrollos de la moderna física matemática.

Entre estos estudiosos tenemos al segoviano Luis Coronel conciudadano y profesor de Domingo de Soto. Como novedad en su tratamiento del movimiento local está la introducción que hace del valor medio de la velocidad en el caso del movimiento uniformemente o diformemente acelerado. Y además de esto se sirve de la regla de los calculadores para aplicarla a los diversos casos del movimiento local y a las distintas ejemplificaciones que propone.

Otro español al que tenemos que hacer alusión es a Juan de Celaya, profesor en la Universidad de París, en cuyos escritos se da la conjunción 
entre la línea de los calculadores y el realismo de los comentadores italianos de Aristóteles. Este escolástico fue profesor de Domingo de Soto y es posible que fuera él quien le influyó para adoptar el esquema de los calculadores de Oxford en su explicación del movimiento local.

En este siglo XVI y dentro de la Península ibérica tenemos que citar a los siguientes autores: Diego Diest y su tratado Quaestiones physicales publicado en la Universidad de Zaragoza en 1551; Diego de Astudillo y sus Quaestiones publicadas en la Universidad de París en 1532; Pedro Margalho y su Physices compendium publicado en Salamanca en 1521 y Domingo de Soto y sus Quaestiones publicadas en Salamanca en 1555.

Lo importante de Domingo de Soto es que vuelve al esquema de una sola variable de Heytesbury, del que da una explicación exhaustiva proponiendo ejemplos para todos los casos; y es con este trasfondo como él asocia la caida de los cuerpos con el movimiento uniformemente diforme, dando a tal expresión el significado preciso de «movimiento uniformemente acelerado en el tiempo», al cual aplica el teorema de la velocidad media del Colegio de Merton.

La introducción del pensamiento de los calculadores en el Colegio Romano de los jesuitas en la ciudad de Roma fue obra sobre todo del español Toledo que enseñó allí filosofía natural el año 1560, importando a dicho Colegio las ideas que había recibido en Salamanca del dominico Soto. En esta tarea otros dos españoles jugaron un papel destacado como es el caso de Benedicto Pereira y Francisco Suárez. Este último enseñó teología en dicho Colegio los años de 1580-1585; y puede servirnos de referencia para entender el papel de mediadores de los jesuitas en el proceso de transformación de la cinemática medieval en la cinemática de los modernos. En sus Disputaciones Metafisicas de año 1597 Suárez reflexiona sobre el tema de la intensión y remisión de las formas aludiendo al concepto de «uniformemente diforme» $y$ nos habla de la velocidad como intensidad del movimiento. El rechaza esta tesis, pero es importante destacar la relevancia que concede al tema de la intensión y remisión de las formas, recogiendo en su manual de las Disputaciones la tradición de este tema tal como se ha desarrollado desde finales de la Edad Media hasta su momento. Este manual de Suárez fue muy importante en la formación del pensamiento moderno. Es un texto, que como toda la obra de Suárez, fue preparado y editado en Salamanca y del cual podemos afirmar que es una muestra clara de la importancia de las ideas presentes en la Universidad de Salamanca en el siglo XVI; ideas que Suárez ha sintetizado en toda su obra tanto filosófica, como jurídica y teológica. Las Disputaciones Metafisicas 
de Suárez escritas y publicadas en Salamanca en 1597, después de su estancia en Roma, son una muestra objetiva de la relevancia del ambiente cultural en la Salamanca del siglo XVI. Un análisis detenido de la Disputación XLVI nos permitiría mostrar la diferencia entre la línea más matemática y la más metafísica en el tratamiento del tema de la intensión y la remisión de las formas; al mismo tiempo que veriamos cómo la síntesis realizada por los jesuitas de las ideas de la tradición y de la modernidad naciente son las que van a conducir al desarrollo de la moderna dinámica tal como será planteada por Leibniz. Aquí nos limitamos a aludir este punto que no podemos desarrollar; y para cuyo desarrollo podemos citar las siguientes referencias que damos en nota ${ }^{23}$.

Como conclusión de todo esto que venimos diciendo a propósito de la física matemática moderna tenemos que decir que Wallace ha fijado muy bien la mediación entre los jesuitas y Galileo, y a él remitimos a aquellos que estén interesados en este punto. Lo que nosotros queremos resaltar es que la tesis de Wallace apoya la tesis de Duhem frente a la de Koyré al poner en cuestión la interpretación que este último hizo de Galileo como un platónico y racionalista, que no tuvo necesidad de recurrir a la experimentación para fundar la «nueva ciencia». Las ideas de Galileo de la «nueva ciencia» están emparentadas con la física matemática de los escolásticos y nominalistas de la Universidad de París del siglo XVI, que a su vez son los importadores y reformuladores de la física matemática de los mertonianos del siglo XIV. Y en este punto de reformulación de las ideas sobre el movimiento y de su transmisión a Italia el dominico Domingo de Soto juega un papel que no puede ser desconocido o minusvalorado tal como ha hecho Koyré.

\section{La matemática de mertonianos y nominalistas y el replanteamiento del conocimiento sensible}

Ciertamente la matemática juega un importante papel en la formulación de la nueva ciencia. Pero la matemática puede entenderse en un sentido platónico e idealista, o en un sentido más aristotélico, como es el caso de la tradición que nosotros estamos considerando aquí. Para ilustrar esto puede servirnos otro de los profesores ibéricos de la Universidad de París: Pedro Sánchez Ciruelo, que aparte de otros libros esribió uno titulado Apotelesmata Astrologiae Chistianae, en el que entabla una discusión con Pico della Mirandola que puede 
servirnos para establecer la diferencia entre el modo platónico y el modo aristotélico de entender la matemática.

Ciruelo, siguiendo a Aristóteles, establece una división de las ciencias de acurdo con las «razones formales» bajo las cuales éstas consideran las cosas. Y así nos habla de la física como ciencia de lo sensible, de la matemática como ciencia de lo imaginario; y de la metafísica como ciencia de lo suprasensible. Esa consideración de la matemática como ciencia de lo imaginario tenemos que entenderla en el sentido de que los «objetos» sobre los que trata no existen en la realidad, sino que se «suponen» o se refieren a objetos reales desde unas determinadas «razones formales», que en el caso de las matemáticas coinciden con las mismas cosas naturales. Lo que aquí Sánchez Ciruelo entiende por forma no es un objeto exterior hacia el que apuntan las matemáticas tal como defendería un platónico, sino un determinado modo de objetivación de la realidad que podemos calificar de hipotético. El mundo de las formas matemáticas es un mundo de ordenación de la realidad y no un mundo de cosas.

La filosofía matemática de Sánchez Ciruelo es acorde con la epistemología aristotélica, que en el caso de las matemáticas podemos interpretar como «creadora de hipótesis». La forma matemática es una «hipótesis» que los matemáticos han sacado de la observación de los objetos reales, que nos permite una visión de la realidad distinta de la de la física y de la de la metafísica. La visión matemática de la realidad es una forma de objetivación de la misma, gracias a la cual descubrimos aspectos de la realidad que de otra forma no podriamos descubrir. Y lo peculiar de la visión matemática de la realidad es que no se queda en la pura contemplación de la misma, como ocurre con la metafísica, sino que busca la aplicación, que es uno de los aspectos fundamentales de la matemática aristotélica; y que es también la peculiaridad de la aplicación que mertonianos y nominalistas hacen de la matemática a la física.

La teoría aristotélica de las ciencias tal como es interpretada por Ciruelo considera que todas las regiones del conocimiento tienen en su base un «medio común» a partir del cual se especifican los distintos campos científicos. El «medio común» de las ciencias del Quadrivium es la cantidad, estudiada por la metafísica, que luego se especifica como número en la aritmética, como extensión en la geometría, como rayo en la perspectiva y como sonido en la música. De acuerdo con esta concepción el número no es objeto de la metafísica, sino de la matemática en cuanto que ciencia que considera el modo de significar de la numeración, que es un modo de ordenar la realidad de acuerdo 
a una forma de objetivación no sensible. Esta teoría de la ciencia encaja muy bien en una teoría de la dialéctica como "nuevo método» con dos direcciones fundamentales: la hipotético matemática y la teoría escolástica de la suposición.

Esta última se cumple de modo paradigmático en lo que los escolásticos llaman «ciencias mixtas», que emplean dos tipos de premisas: una que suele ser una tesis y otra que suele ser una hipótesis, que muchas veces es una definición que no necesita ser probada, sino que basta con que se suponga su verdad. Esta teoría de la suposición es la que va a permitir a Soto articular física y matemática en su interpretación de los libros físicos de Aristóteles a los que nos hemos referido anteriormente.

La primera de las direcciones aquí aludidas y que es la que tiene que ver con la línea hipotética de la matemática nos puede permitir ver cómo en la época del Renacimiento se genera una nueva concepción de la naturaleza, que tiene en su base la «fantasía sensible» tal como la misma ha sido tematizada por la tradición aristotélica de esta época. Se trata en definitiva de una reivindicación del soñar íntimamente asociada a la ciencia en el aspecto epistemológico que podemos unir con la moderna teoría de las hipótesis, en la que experimento y razón se dan unidos en el proceso circular del conocimiento. Se parte de una hipótesis (forma sacada de la observación de los fenómenos) para llegar nuevamente al fenómeno que confirme la hipótesis. Y este mismo proceso circular se da también en el uso que los físicos naturales del final de la Edad Media y principio del Renacimiento hacen del experiemento: se parte de un fenómeno (experiencia) para llegar a descubrir las razones (razón) que gobiernan a ese fenómeno y hacen de él un «caso» del proceso necesario de la naturaleza.

En este punto podemos apreciar una diferencia profunda entre el planteamiento platónico y aristotélico en cuanto a la interpretación del conocimiento hipotético. En el $\mathrm{n}^{0}$ 533c de La República Platón contrapone el camino del dialéctico y del matemático; y califica el arte de la geometría como un conocimiento que no llega a la perfección del conocimiento del dialéctico, por el hecho de que en lugar de contemplar la esencia, que es lo propio de la dialéctica, se dedica a «soñar» hipotéticamente, manteniéndose por lo tanto en el terreno de la opinión y sin llegar al plano de la ciencia, en el que no puede darse ningún tipo de sueño. Esto supone una valoración negativa del conocimiento hipotético por parte de Platón.

Frente a este planteamiento platónico encontramos en la epistemología de Aristóteles una valoración muy positiva del conocimiento 
hipotético practicado por las matemáticas. Para entender el planteamiento positivo que Aristóteles hace de las hipótesis tenemos que referirnos a su teoría de la imaginación. $\mathrm{Y}$ al hacerlo podemos decir que en Aristóteles pueden distinguirse dos tipos de imaginación: una dependiente de los sentidos y que también poseen algunos animales; y otra ligada a lo que Aristóteles denomina fantasía y que es propia del hombre. De esta última dice Aristóteles en el c. $3^{\circ}$ del libro III del De anima: "Podemos imaginar a voluntad - es posible, en efecto, crear ficciones y contemplarlas como hacen los que ordenan las ideas mnemotécnicamente creando imágenes» ${ }^{24}$. Esta caracterización de la imaginación como unida a la voluntad es la que le permite a Aristóteles distinguir entre la reminiscancia, que es más mecánica, y el recuerdo, que es más voluntario, tal como se desprende del siguiente texto: «Pues recordar es tener en uno mismo la capacidad de provocar un proceso, y ello de manera que uno lo experimenta por sí mismo y por los procesos que posee, como se ha dicho. Es preciso, pues, tomar un punto de partida. Por ello parece que a veces se rememora a partir de lugares» ${ }^{25}$. Hipotetizar para Aristóteles es imaginar la realidad a base de formas sacadas de las cosas. Y esas formas no son copias de las cosas de que están sacadas, sino figuraciones de la realidad que nos permiten ver esta realidad como no podemos hacerlo sirviéndonos exclusivamente de los sentidos. "En efecto, de igual modo que la figura dibujada en un cuadro es una figura y una imagen $-y$ aunque es una y la misma es ambas cosas, pero la esencia no es la misma para ambas y es posible contemplarla con la mente como figura y como imagen-, así también es preciso considerar la imagen que hay en nosotros como tal imagen por sí misma, al mismo tiempo que como imagen de otra cosa. De este modo,en la medida en que se la considere por sí misma, es un objeto de contemplación o una imagen, pero, en la medida en que se la considere como otra cosa, es como una copia y un recordatorio» ${ }^{26}$. Lo propio de las matemáticas es figurar hipotéticamente la realidad por medio de la captación de una forma sacada de esa misma realidad. Y en este caso las figuraciones de la realidad se refieren a la realidad, pero no hay que confundirlas con entidades reales tal como hacen los platónicos. Para Aristóteles los números no son entidades reales, sino imaginarias, pero que nos permiten ver una dimensión de la realidad que no es posible desde el punto de vista de los sentidos. Es decir, que el aristotelismo lleva consigo una consideración de la matemática, que hace de esta un saber concreto y aplicable a la realidad. $\mathrm{Y}$ esto es precisamente lo que van a explotar algunos escolásticos de finales de la Edad media así como algunos 
humanistas haciendo pasar a primer lugar y como objeto fundamental de su estudio la física matemática entendida como matemática aplicada al fenómeno del movimiento.

\section{Los sensibles comunes de la epistemologia aristotélica}

En la epistemología aristotélica nos encontramos con una teoría de los «sensibles comunes», que reviste una notable importancia para los temas que venimos tratando y por ello debe ocupar ahora nuestra atención. En su tratado Acerca del alma nos habla Aristóteles de una facultad central, que juega una función unificadora en relación con las distintas fuentes sensibles a través de las cuales llega a nosotros la experiencia del mundo. Esa facultad central no está bien fijada y ha recibido distintos nombres según los comentaristas de Aristóteles. Unos colocan esta facultad central en el plano de los sentidos y nos hablan de un sentido común, que es el que unifica a los distintos sentidos particulares y el que a su vez nos permite captar los sensibles comunes; aunque si nos fijamos en el texto De Anima de Aristóteles: 425a y ss. parece dar a entender que no hay un tal sentido común perceptor propio de los sensibles comunes, sino que estos se perciben como resultado de la sensación común a los distintos sentidos, sensación común que tendría su fundamento en el hegemonikon del alma. Podemos afirmar entonces que es el alma la que percibe los sensibles comunes gracias a una facultad mediadora entre los sentidos y la razón. Y esa facultad mediadora no es otra que la imaginación, que en el caso del recuerdo trabaja de modo inferencial haciendo que percibamos no sólo lo individual, sino también lo común, que podemos relacionar con lo imaginable. Percibimos lo común gracias a la imaginación que nos permite apreciar un aspecto de la realidad sensible que no se nos da en los sentidos particulares ${ }^{27}$. Entre esos sensibles comunes Aristóteles cita la magnitud, el movimiento, el número y el tiempo. Para Aristóteles estos sensibles comunes no existen separados como pensaran platónicos y pitagóricos, sino encarnados en las cosas físicas tal como da a entender muy bien en la Física 194a y ss. Por eso para él los objetos de las matemáticas no existen como algo separado, sino como aspectos de las cosas, que nuestra imaginación separa de la materia para mejor conocerlos. Y por eso Aristóteles nos habla de dos tipos de ciencias matemáticas: las matemáticas puras como la aritmética y la geometría que estudian sus objetos como «separados» de la materia; y las matemáticas mixtas como la óptica y la astronomía que estudian sus objetos como «encarnados» en la materia. Esta interpretación aris- 
totélica de las matemáticas es de una gran relevancia para los aspectos que estamos tratando en nuestro artículo. Pero vamos a fijar nuestra atención en un punto en el que confluyen ciencia y arte; y ese punto es la teoría del diseño que es de una gran relevancia en la época del manierismo, que es la que se corresponde con nuestro plateresco.

Podemos relacionar la teoría del diseño con la teoría del ingenio, que supone un cierto naturalismo en la interpretación de las facultades naturales de que está dotado el artista o el científico. En los Analíticos Posteriores nos habla Aristóteles del que él denomina «universal commensurado»; y dice de él que es presioso, porque pone en evidencia la causa ${ }^{28}$. En esa misma obra, en el capítulo último del libro $1^{\circ}$, habla de la perspicacia o agudeza y la define como «la facultad de encontrar el término medio instantáneamente» ${ }^{29}$. Esa perspicacia o agudeza será también lo propio del poeta en el caso de las metáforas, cuyo uso apropiado no se puede aprender de otro y es índice de «natural ingenio», "porque la buena y bella metáfora es contemplación de semejanzas» ${ }^{30}$. Y lo mismo que decimos de la bella y buena metáfora podemos decir de los sensibles comunes y del universal commensurado. Es decir, que lo que es la metáfora al poeta es el universal commensurado al científico y el diseño al artista. Todos ellos tienen en común la agudeza o perspicacia para aprehender lo semejante a distintos géneros. Aquí reside precisamente la peculiaridad de la teoría aristotélica de la proporción, que tal como es aludida en los Tópicos puede servir de base común a magnitudes y números ${ }^{31}$; mientras que en los Analíticos Posteriores parecía defender que tiene que ser algo distinto en el caso de la magnitud y en el caso del número ${ }^{32}$. Lo que podemos concluir de todas esta reflexiones es que la teoría aristotélica de la agudeza o perspicacia tiene como objeto la percepción de lo común a elementos de distintos géneros, o lo que también podriamos decir la percepción del término medio tal como es interpretada esa noción en la Etica a Nicómaco, en la que Aristóteles compara la prudencia, fruto de la experiencia, con el saber matemático, fruto de la abstracción; $\mathrm{y}$ nos dice que un joven puede ser un buen matemático, pero no un buen físico, como tampoco podemos calificarle de prudente. Ahí distingue Aristóteles entre deliberación y buen tino y nos dice de este último que es rápido y no necesita razonar como es el caso de la deliberación ${ }^{33}$.

\section{Armonia y proporcionalidad.}

El estudio de la teoría de la proporción puede servirnos para apreciar la conjunción entre arte y ciencia en la Salamanca del siglo XVI, en 
cuya segunda mitad nos enontramos con el arquitecto Rodrigo Gil de Hontañón que va a elaborar un manuscrito que será refundido por Simón García en 1681-1683 con el título de Compendio de arquitectura.

Como el mismo Simón García nos dice la mayor parte del manuscrito que él deja listo para la imprenta en 1681-1683 es fruto del trabajo de Rodrigo Gil de Hontañón, que trabajó como arquitecto en Salamanca en la segunda mitad del siglo XVI. Podemos, pues, leer el manuscrito como un "tratado" de arquitectura que sintetiza el saber sobre esa ciencia tal como la misma era concebida por un arquitecto en la segunda mitad del siglo XVI. Es cierto que es difícil determinar lo que corresponde a Gil de Hontañón y lo que es propio de Simón García; pero sí que podemos mantener que el núcleo orginal y amplio del tratado es de mano de Rodrigo Gil de Hontañón; y por lo mismo podemos considerarlo como un ejemplo claro del saber de la arquitectura en la segunda mitad del siglo XVI, que no solamente tiene pretensiones prácticas, sino también teóricas. $\mathrm{Y}$ por eso su análisis nos permite descubrir el modo como Gil de Hontañón ha sintetizado el estado de la ciencia en la ciudad de Salamanca en esa segunda mitad del siglo XVI en torno a la arquitectura.

En el capítulo VII trata de los órdenes de la arquitectura y al hacerlo va entretejiendo una serie de reflexiones sobre la simetría, la arquitectura como ciencia y una extensa digresión sobre la diferencia entre oficio, arte y ciencia. Defiende el carácter científico de la arquitectura y 'sace de ella un arte liberal por la razón de que lo más importante de la misma es obra del entendimiento. Y caracteriza la simetría como una «numeración conmensurable» siguiendo a Plinio y a Vitrubio, y la define de la siguiente forma: «Es una proporcionada medida, partida numeralmente en dibersas quantidades, $\mathrm{Y}$ como dice euclides, niguno la puede alcancar, si no fuere exercitado, y erudito aritmético, y precisamente la divina Regla de la proporción humana, no tubiere bien entendida» ${ }^{34}$.

Uno de los aspectos más importantes del texto es precisamente esta caracterización de la simetría. En él podemos ver dos formas de tratar la proporción. Por un lado la forma antropométrica siguiendo la tradición antigua, medieval y renacentista; y por otro lado la forma geométrica, que es quizá la más importante del tratado. Una parte importante del tratado está dedicada a la proporción matemática recogiendo en buena medida el tratamiento que de la teoría de la proporción hicieran matemáticos y filósofos a lo largo del siglo XVI y XVII. A este respecto es importante el capítulo 67 en el que hace una especie de historia del tratamiento de la proporción distinguiendo 
perfectamente los siguientes aspectos. En primer lugar el tratamiento pitagórico de la proporción distinguiendo entre proporción aritmética, geométrica y armónica. En segundo lugar ditingue ese tratamiento de la proporción del que podemos llamar tratamiento matemático de la proporción, que es un tratamiento más complejo y sutil y que se apoya en la relación recíproca entre dos o más fracciones, gracias a la presencia de un término común, que es el que posibilita establecer las distintas relaciones, que ya no se reducen a tres, sino que son muchas más que se han ido acumuando en la tradición de la historia de la matemática tal como nos dice el mismo autor del tratado, que escribe lo siguiente: "Y otros filósofos, desde tiempo de pitágoras, asta de platón, no abian considerado, más de 3 . especies, $\mathrm{O}$ diferencias de proporcionalidades que por otro nombre, dijeron mediaciones, que eran las que se dicen, arismética, Geométrica, y armónica, de que tratamos en el capítulo 62. Y este número duró, hasta el tiempo de Aritotiles, después los que a estos sucedieron, añadieron otras 3 . Y después sobre estos, añadió boecio otros 4. que fueron 10. y jordano, una, como refiere jacobo fabro, que todas son 11. las 3. primeras que son, proporcionalidad, arismética, Geométrica,y harmónica, la imbentó pitágoras, $\mathrm{O}$ en su tiempo, las cuatro que le siguen, las añadió nicomacho, las otras 3 . añadió Boecio, Y jordano añadió la última» ${ }^{35}$. Pensamos que esta historia está tomada de Jacobo Fabro Estapulense, a quien cita en el texto, que es un filósofo francés del siglo XVI, que tradujo la Aritmética de Nicómaco de Gerasa. Curiosamente Juan Martínez Silíceo, que el año 1517 publicó en París un Ars Arithmetica y que fue discípulo de Jacobo Fabro escribe en ese libro lo siguiente: «Esta última media es un añadido de Jordano en el libro X de sus Elementos a las 10 medias que expuso Boecio en el libro II de su Aritmética» ${ }^{36}$. Martínez Silíceo fue uno de los profesores de filosofía natural de la Universidad de Salamanca en la primera mitad del siglo XVI; y en el "tratado cuarto" del citado libro en el que trata de las relaciones entre los números escribe: «Si un número se compara con otro número, surge ahí una relación que llamamos proporción. $\mathrm{Y}$ si una relación se compara con otra relación y una proporción con otra proporción tendremos inmediatamente una media o una proporcionalidad» ${ }^{37}$. Esta misma idea la encontramos en el autor del «Compendio de Arquitectura» que escribe: "Algunos llaman proporción, a lo que aquí se dice proporcionalidad, Y proporcionalidad, a lo que los otros llaman proporción. Yo llamo en esta obra, proporción, a la comparación que se hace de un número a otro, y proporcionalidad, a la comparación hecha de una proporción, a otra» ${ }^{38}$. 
Esta teoría de la proporcionalidad matemática es aplicada por Rodrigo Gil de Hontañón a la construcción de la Catedral nueva de Salamanca tal como nos dice el mismo Simón García en el capítulo 12 del Comprendio. «La fábrica de la yglesia nueva es, Gótica tracada, Proseguida, y aprovada, por los mejores architectos, que en aquella edad avia.hordenola juan Gil de Hontañón, y executola, Rodrigo Gil su hijo (de quien es lo más de este compendio por a ber venido a mis manos, un manuscrito suio)» ${ }^{39}$. Y a continuación nos da las medidas: «Repartieron el templo en esta manera de largo, 378. pies, sin los Gruesos de las paredes; de ancho, 181. sin los gruesos, la nave maior 50. Y cada, lateral a 37., y medio las ornacinas a 28. desde los pies, asta el crucero 5 a 37 y medio de largo, al crucero 50. que es cuadrado, a la capilla maior 75 . que es largo de dos capillas, al trascoro $37 \mathrm{y}$ medio a la ornacina del trascoro, 28. los pilares, a 10. de diámetro, los cuatro del crucero a 12.de diámetro; Dieronle de alto a la nave maior 130. de claro a los colaterales 88 . de claro, a las ornacinas, 54. pies de claro» ${ }^{40}$.

En el texto podemos apreciar el abandono de la proporción antropométrica y la asumción de la proporción matemática. Este predominio de un tipo de proporción sobre otra es la expresión clara de la transformación que está experimentándose en la idea de espacio, que en nuestro ejemplo está concretada en la de espacio arquitectónico. Se abandona la idea de espacio caracterizada a partir de una teoría del lugar y se pasa a una idea de espacio entendida como una función ordenadora de la realidad, que es la que va a conducir al principio general de la homogeneidad del espacio. La física aristotelica distinguia los espacios como algo heterogéneo de acuerdo con los distintos lugares naturales; mientras que la nueva física que está surgiendo en el Renacimiento va a homogeneizar los espacios y a hacer de este concepto un concepto universal regido por leyes matemáticas, cuyo funcionamiento es el mismo ya se trate del espacio de la tierra, o del espacio cósmico. En el Compendio que aquí hemos analizado hemos visto como el concepto de simetría se interpreta fundamentalmente en términos numéricos haciendo posible un concepto de «espacio sistema» alternativo al concepto de "espacio agregado» característico de la física antigua y medieval.

\section{Conclusión}

Podemos hablar de cuatro ámbitos en los que vemos transformarse en la época del Renacimiento la idea de espacio: en el ámbito de la 
cosmografia con su correspondiente representación de los lugares en los mapas; en el ámbito de la filosofía natural con su nueva interpretación del movimiento local; en el ámbito de la arquitectura con su nuevo concepto de espacio sistema; y finalmente en el ámbito de la cosmología con su transformación del espacio supralunar en un espacio homogéneo al terrestre, cosa que ocurre con la nueva interpretación de los cometas. Ejemplos de estas cuatro transformaciones encontramos en la Salamanca de los siglos XV y XVI. Aquí hemos analizado dos de ellas: la segunda y la tercera de las aquí citadas.

Vamos a concluir nuestro artículo con una reflexión general que nos permita dar unidad a las ideas que hemos expuesto en él. Esas ideas las podemos sintetizar en el cambio que tiene lugar entre una concepción del espacio como «espacio agregado» y su sustitución por la de un espacio como "espacio sistema». Esta sustitución la hemos ejemplificado en el caso del tratamiento del movimiento local y en el caso de la arquitectura fijando nuestra atención en autores y textos en los que se cumple ese cambio. Esto nos permite afirmar que la Salamanca del siglo XVI es una pequeña ciudad en la que circulan algunas de las ideas que van a conducir a la moderna revolución del espacio tal como la veremos cristalizar en la cosmovisión física de Galileo en el siglo XVII. En el fondo de esa revolución y cambio subyace una concepción común que se concreta en la teoría de la proporción, que es una teoría matemática que se aplica tanto en el caso de la física (explicación del movimiento local), como en el caso de la arquitectura (teoría de la simetria).

La teoría de la proporción fue elaborada por el pitagorismo para explicar filosóficamente la relación entre lo inteligible y la forma sensible. La teoría de la proporción es la noción suprema de toda la aritmética pitagórica gracias a la cual se puede acceder al estudio de la naturaleza física (tierra), de la música, de la astronomía (naturaleza cósmica) y de la geometría. Platón en su filosofía asumirá esta teoría pitagórica de la proprción y afirmará que ella es la causa de todo lo que es armónico, racional y ordenado. Esta teoría pitagórica de la proporción fue desarrollada por dos tradiciones. Una de ellas es la tradición de los matemáticos en la que el eslabón fundamental va a ser Euclides y los posteriores comentarios a algunos de los libros de sus Elementos. La otra tradición es la de los platónicos, que es una tradición más metafísica. En esta tradición nos encontramos sobre todo con los comentadores del Timeo entre los que podemos citar a Nicómaco, Teón y Jámblico. Esta tradición, siguiendo la metafísica pitagórica, va a defender que los números representan la esencia inteligible de 
la realidad; y esa esencia se expresa en formas visibles tanto en el campo del arte como de la ciencia. Una aplicación de esta teoría metafísica de las proporciones es la consideración del cuerpo humano como paradigma de medida tal como va a hacer, por ejemplo, Leonardo en su famosa representación del cuerpo humano como modelo antropométrico.

Estas dos tradiciones comparten en común una matematización del universo, que tiene como objetivo fundamental lo que algunos teóricos de la historia de la ciencia han calificado como «salvar las apariencias». Pero ese «salvar las apariencias» va a ser interpretado de forma distinta en el caso de las dos tradiciones. La tradición de los matemáticos y en concreto de Euclides y sus comentadores (en esta tradición es en la que hay que colocar a Aristóteles) va a dar lugar en la modernidad al «método experimental» entendido como algo más complejo que la simple observación; ya que el fenómeno que se pretende salvar está puesto por el observador (Aristóteles diría que ha sido abstraido de la realidad física) y sólo puede ser comprendido en su relevancia si lo insertamos dentro de una estructura teórica. Esta tradición la podemos relacionar con el realismo experimental de Aristóteles tal como este autor da a entender en su Metafisica y en el De Coelo. Esta es la tradición que va a llevar a los escolásticos del siglo XVI y en concreto a Domingo de Soto a aplicar la matemática a la física de la naturaleza con los resultados que hemos visto en lo anteriormente dicho.

La tradición pitagórico-platónica, en cambio, va a estar más preocupada por la elaboración de una metafísica y se va a preocupar menos de la aplicación de la matemática a la realidad. Es decir, se trata de una tradición en la que la matemática se eleva al nivel de la metafísica y hace del número la esencia de la realidad.

Por lo tanto podemos afirmar que la interpretación aristotélica de la matemática está más próxima de la revolución científica de los modernos (léase Galileo) que la interpretación pitagórico-platónica, a pesar de que Koyre haya defendido lo contrario y en consecuencia no haya sabido apreciar el llamado «enigma de Soto». Lo cierto es que como consecuencia de la aplicación de la matemática a la realidad las artes liberales conflueyn con las artes mecánicas y todas ellas pasan a constituir en conjunto el nuevo corpus armónico y sistemático del saber moderno, del que podemos afirmar que tiene como raiz común a los distintos tipos de artes una teoría matemática de la proporción interpretada desde el punto de vista de la «igualdad», como se ve muy claro en el caso de la igualdad armónica. Si podemos hablar de una proporción armónica es porque entre los elementos de la misma 
(que pueden pertenecer a campos muy diversos) se da una relación de igualdad. Esto es lo que quería dar a entender Simón García cuando nos decía, como hemos visto en el interior del artículo, que hay que distinguir entre proporción y proporcionalidad. La llamada proporción armónica se construye gracias a un conjunto de relaciones de igualdad; y ahí reside la esencia de la proporcionalidad, que también podemos encontrar en el lenguaje como es el caso de la metáfora tal como hemos visto al hablar de Aristóterles. La esencia de la proporcinalidad se encuentra en aquello que es "común» a géneros diferentes; y ese elemento común se puede expresar tanto en términos numéricos (fórmulas matemáticas) como linguísticos (metáforas). Estamos ante lo que los aristotélicos denominan los «sensibles comunes» entre los cuales colocan el movimiento. Y. para la explicación de esos sensibles comunes los aristotélicos van a servirse de la matemática como un saber creador de hipótesis, que nos permite explicar aspectos de la realidad física, que no podemos hacer con la simple descripción de esa realidad. Esto se ve muy claro en el caso de Aristóteles en el ejemplo que pone de la óptica y la astronomía en Física 194a, donde explica muy bien cómo entiende él el "salvar las apariencias»: como un pasar de la apariencia a la realidad "more geométrico", que nosotros podemos interpretar como por medio de un razonamiento («more geométrico»), que no es otra cosa que la aplicación de la matemática a la realidad tal como hace el «método experimental» de los modernos muy bien ejemplificado por Galileo y los dialécticos de París entre los cuales podemos contar a Domingo de Soto.

Las matemáticas ocupan una posición intermedia entre la ciencia natural y la divina y pueden alcanzar el primer grado de certeza a causa de que dependen de sus propios principios y fundamentos, que son inviolables y manifiestos a todos. Esta excelencia del saber matemático es la que hace de este saber el mejor camino (método) hacia cualquier otro tipo de saber. Este es el planteamiento que Sánchez Ciruelo hace en su Prefacio al Uberrimum sphere mundi commentum.La suprema dignidad y el valor supremo de la ciencia reside en que nos conduce al conocimiento de lo divino. Por esta razón las matemáticas, que poseen la máxima certeza, son las ciencias dignas de la mayor estima. Y para confirmar esta idea cita a Aristóteles en los siguientes términos: "En el sexto y en el undécimo de la Metafisica, el Filósofo colma de alabanzas a las matemáticas: ya que dice que el género de las matemáticas es ciertamente el mejor, y las llama en verdad ciencias por antonomasia. $\mathrm{Y}$ en el primero de los Posteriores dice: «Las ciencias matemáticas son, con relación al entendimeinto, 
como el ver». Hasta tal punto es, en efecto, clara y evidente la noción que de las cosas transmiten, que con derecho merecen llamarse intuición y visión. $\mathrm{Y}$ en el sexto de los Tópicos afirma que en las matemáticas las mismas cosas nos son más conocidas... Donde quiera que el filósofo trata de declarar el modo científico demostrativo, se vuelve al ejemplo de las ciencias matemáticas, lo que es fácil de ver en sus libros lógicos, físicos y metafísicos» ${ }^{41}$. Junto a esta caracterización de las matemáticas como modelo de conocimiento claro y cierto, presenta Ciruelo la división de las ciencias matemáticas. Siguiendo a Aristóteles en su Física: las ciencias matemáticas se dividen en puras (aritmética y geometría) y mixtas (música, perspectiva y astrología). Las puras son totalmente ciertas, porque utilizan la demostración científica, y se ocupan de cosas simples como son los números, las líneas y las superficies. Las mixtas, aun siendo demostrativas, admiten opiniones.

Lo propio de la matemática es el ser un arte analítico que consiste en disponer adecuadamente la progresión en lo homogéneo para establecer el orden adecuado que nos conduzca a la verdad. Y en esta cuestión del orden es muy importante la dialéctica. En su discusión con Pico de la Mirándola acerca de la astrología Ciruelo establece con nitidez la diferencia entre retórica y dialéctica. El se considera un dialéctico, mientras que Pico es un retórico y por eso no entiende los aspectos epistemológicos de las ciencias. Para Ciruelo la división de las ciencias hay que hacerla desde el punto de vista de las razones formales bajo las cuales aquellas consideran las cosas; y no desde el punto de vista de las cosas mismas como hace Pico. La matemática es un tipo de idealización de la realidad, que nos permite una visión de ésta distinta de la de la física y la metafísica. La matemática ve la realidad bajo una razón formal y no se queda en la contemplación de esa realidad, sino que busca una aplicación de su conocimiento. En este punto es muy interesante la interpretación que Ciruelo hace de las distintas regiones del conocimiento. Todas esas regiones, a las que corresponden las distintas ciencias, tienen como base un «medio común» que es la cantidad, que como tal es objeto de la metafísica o filosofía primera. Esta es la idea que le va a permitir a Descartes hablar de una «mathesis» como una especie de filosofía primera, que es común a la artimética y a la geometría y que funda un saber universal. La importancia de las matemáticas para Ciruelo está en que proporciona a las ciencias un lenguaje común, que por mediación de la dialéctica puede conducir a la construcción de un método universal. Esto es precisamente lo que hará Descartes en el siglo XVII. 


\section{Cirilo Flórez Miguel}

\section{Notas}

1 La ciudad de Salamanca ha sido objeto de varios estudios. Podemos citar en primer lugar el de Gil González Dávila: Historia de las Antiguedades de la ciudad de Salamanca. Salamanca, 1606. Existe una edición facsímil de la Diputación de Salamanca, Salamanca, 1994, muy bien prologada por B Cuart. En el capítulo segundo de esa obra, en la página seis del original de 1606 Gil González Dávila dice que la estructura de la ciudad es circular. Esta caracterización como "ciudad circular" se adecua al modelo ideal de ciudad tal como la misma fue pensada por los antiguos y renacentistas. Gil González Dávila recoge esa idea de la ciudad ideal y se la aplica a Salamanca. La gran reforma que la ciudad va a experimentar en el siglo XVI hará que la ciudad de Salamanca haga honor a esa caracterización de ciudad ideal que a principios del siglo XVII Gil González Dávila le atribuye. Esa caracterización nos sirve muy bien para mostrar el cambio que se produce en la ciudad desde un "espacio agregado" fruto de la mentalidad medieval hacia un «espacio sistema" acorde con la nueva mentalidad renacentista, que es precisamente lo que va a acabar "peculiarizando" a la ciudad de Salamanca.

2 La idea que inspira nuestro planteamiento está tomada de E. Cassirer: Individuo y cosmos en la filosofía del Renacimiento.Trad. A. Bixio, Emecé, Buenos Aires, 1951. En este texto Cassirer estudia entre otras cosas la transformación que ocurre en la ciencia y en el arte del Renacimiento en Europa. Ese es el planteamiento que nosotros vamos a aplicar a la ciudad del Salamanca del siglo XVI, porque pensamos que es una buena ejemplificación de las deas de Cassirer.

3 López Benito, C. I.: La nobleza salmantina ante la vida y la muerte (1476-1535). Diputación de Salamanca, Salamanca, 1991.

4 Ibidem, 59.

5 Una de las características más claras de la mentalidad moderna y renacentista es precisamente la afirmación de la individualidad. Esa característica se da en el caso de la "casa-palacio" de la élite nobiliaria y caballeresca; así como en las «impresas" de los hombres de letras, que es una segunda élite de la ciudad de Salamanca. En el caso de los hombres de letras podemos apreciarlo en las "portadas" de los libros, que juegan un papel similar a las portadas de las casas-palacio. En uno y otro caso se trata de la afirmación de la propia individualidad ante el otro. Una individualidad más de familia en el caso de los nobles y caballeros; y una individualidad más personal en el caso de los hombres de letras. Podemos poner como ejemplo tres "impresas" de hombres de letras: la de Pedro Sánchez Ciruelo, la de Juan Martínez Silíceo y la de Fray Luis de León.

6 Beltrán de Heredia,V. : Accidentada y efimera aparición del nominalismo en Salamanca. Ciencia tomista, LXII, Salamanca, 1942.

7 Villoslada, R.G.: La Universidad de París durante los estudios de Francisco de Vitoria. Roma, 1938. Pueden consultarse las páginas 127-128 en las que se refiere a J. Mair.

8 Vives, L.: In Pseudodialécticos. Ed de Mayans, Valencia, 1782-1790, III, 63.

9 Caroti, S. y Souffrin, P.: La nouvelle Physique du XIV siecle. Olschki, Firenze, 1997.

10 El título completo del libro es: Calculatoris Swiset Anglici sublime et prope divinum opus in lucem recenter emissum cura atque diligentia Philosophi Silicei. Impreso en la imprenta de Juan de Torres, Salamanca, 1520. Consta de 16 capítulos en los que se expone la teoría de la intensión y la remisión de las formas. 


\section{La ciudad de Salamanca en el siglo XVI: La conjunción...}

11 Oresme, N.: Tractatus de configurationibus qualitatum et motuum. Edición de M Claget, University of Wisconsin Press, Madison, 1968, 409-411.

12 La importancia de la "dialéctica" en el sigl XVI es extrarodinaria. Podemos decir que se trata del tipo de saber peculiar de ese siglo, en el que se editan una multitud de libros que tienen en su título dicho nombre. Quizá el sentido más común de este término en ese siglo XVI es el de "método". La dialéctica es un método de acceso al saber o conocimiento. A la hora de especificar ese método unos van a poner el acento en una teoría de la argumentación; mientras que otros van a poner el acento en el componente matemático del mismo. Este último aspecto es el que se ha hecho más famoso por el hecho de que es lo que hará Descartes al escribir su famoso Discurso del método, que todo estudioso de la filosofía propone como el paradigma de lo que será el modo moderno del filosofar. Pero lo cierto es que el elemento común a uno y otro planteamiento viene dado por el nombre genérico de "dialéctica"; y como una ejemplificación famosa de todo esto se habla de los «dialécticos de París» por ser la Universidad de más renombre en relación con este punto. Puede consultarse: A. Robinet: Aux sources de l'esprit cartésien. Vrin, París, 1996.

13 Margalho, P.: Escolos em ambas dos logicas. Trad.: Pinto de Meneses. Instituto de alta Cultura, Lisboa, 1965, 83.

14 Este elogio de la Universidad de Salamanca que Margalho pone en su libro de Lógica va a ser recogido por Cervantes casi un siglo después cuando en el capítulo XVIII de la segunda parte pone en boca de don Quijote las siguientes palabras dirigidas al hijo del caballero de Miranda: «Mereceis estar laureado... por las Academias de Atenas, si hoy vivieran, y por las que hoy viven de París, Bolonia y Salamanca".

15 Gómez Pereira: Antoniana Margarita. Trad. J.L. Barreiro y C. Souto. Universidad de Santiago de Compostela, Santiago de Compostela, 1999.

16 Ibidem, 277.

17 Domingo de Soto: Super octo libros phisicorum Aristotelis Quaestiones. F ${ }^{\circ}$ 92v., Portonaris, Salamanca, 1572. Es la tercera edición.

18 Duhem, P.: Études sur Leonard de Vinci. Hermann, Paris, 1906-1913.

19 Koyré, A.: The enigma of Domingo de Soto. En AA. VV.: Hystory of Science. Basic Books, New York, 1964, II, 94-95.

20 Wallace, W.A.: Galileo and his Sources. Princeton University Press, Princeton, 1984. Es también relevante el artículo de este mismo autor: Duhem and Koyré on Domingo de Soto. Revista Synthesis, 1990.

21 Bardwardine,T.: Tractatus de proportionibus velocitatum in motibus.

Heytesbury,W.: Regulae solvendi sophismata.

Swineshead, R.: De motu. Liber calculationum. Tractatus de motu locali.

22 Este planteamiento está tomado del artículo de Giovanni de Iuliis: "Uniformiter difformis motus». Domingo de Soto e la nascita de la fisica moderna. En Ada Lamacchia: La Filosofía nel Siglo de Oro.Studi sul tardo Rinascimento spagnolo. Levante Editori, Bari, 1995, pags. 169-211.

23 Suárez,F.: Disputaciones Metafisicas. Trad. S. Rábade y otros. Gredos, Madrid, 1964.

Lewis,C.: The Merton tradition and kinematics in late sixteenth and early seventeeth century Italy. Antenore, Padova, 1980.

Gueroult, M.: Leibniz.Dynamique et Metaphysique. Aubier, Paris, 1967.

24 Aristóteles: Acerca del alma. Trad. T. Calvo, Planeta-Agostini, Barcelona, 1995, 141.

25 Aristóteles: Acerca de la memoria y la reminiscencia, En Tratados breves de historia natual. Trad. A. Bernabé Pajares, Planeta-Agostini, Barcelosna, 1996, 129. 


\section{Cirilo Flórez Miguel}

26 Ibidem, 122.

27 Aristóteles: Acerca de la memoria y la reminiscencia. En Tratados breves. Trad. A. Bernabé. Planeta-Agostini, Madrid, 1996.

28 Aristóteles: Analíticos Posteriores, 88a.

29 Ibidem, 89b.

30 Aristóteles: Poética, 1459a.

31 Arstóteles: Tópicos, 158b.

32 Aristóteles: Analíticos posteriores, 74a.

33 Aristóteles: Etica a Nicómaco, 1142a-b.

34 Garcia, Simón: Compendio de Architectura y Simetria de los Templos. Colegio oficial de arquitectos de Valladolid, Valladolid, 1991, 77.

35 Ibidem, 169.

36 Martínez Silíceo,J.: Ars Arithmetica. E. de E. Sánchez Salor, Universidad de Extremadura, Cáceres, 1996, 128.

37 Ibidem, 123.

38 García, Simón: Compendio, 168.

39 Ibidem, 91-92.

40 Ibidem, 92.

41 Sánchez Ciruelo, P.: Prefacio al Uberrimum mundi commentum, en Flórez, C. y otros: Pedro Sánchez Ciruelo: una enciclopedia humanista del saber. Caja Salamanca, Salamanca, 1990, 89-91. 